\title{
APLICANDO A CULTURA MAKER NO CENÁRIO DE ISOLAMENTO SOCIAL
}

Vinícius Veridiano Miranda-revibe2010@hotmail.com

Guilherme Quirino de Andrade - guilhermeqda@gmail.com

Igor Inácio Cirino - igor_inacio@windowslive.com

Gabriella Castro Barbosa Costa Dalpra-gabriella@cefetmg.br

José Geraldo Ribeiro Júnior - junior@leopoldina.cefetmg.br

Lindolpho Oliveira de Araújo Júnior - lindolpho@cefetmg.br

Centro Federal de Educação Tecnológica de Minas Gerais - CEFET-MG - Unidade Leopoldina

Rua José Peres, 558, Centro

36700-000 - Leopoldina - MG

Resumo: Este trabalho apresenta um estudo de caso acerca das ações adotadas pelo Projeto de Extensão Mundo Maker do CEFET-MG, diante do cenário de isolamento social. O Mundo Maker tem por objetivo a popularização da ciência e da tecnologia associadas a nova era digital na sociedade, com foco em estudantes do ensino fundamental e médio, seguindo a filosofia da cultura maker. Dentre as ações do projeto, destacam-se a realização de minicursos que exploram conceitos de lógica, programação básica e ciência, utilizando uma abordagem prática, além de elaborar um acompanhamento avaliativo diversificado. E, devido à pandemia de COVID-19, o Mundo Maker precisou transferir suas ações, inicialmente planejadas para o formato presencial, para o modelo de um ensino remoto. Neste trabalho, será exposto a origem dessa cultura que inspirou o projeto, bem como a sua relevância para integralização do ensino, não só para o ensino regular, mas também para cursos de graduação. Também apresenta um relato das metodologias abordadas para ofertar os minicursos em um ambiente virtual com a mesma qualidade do formato presencial, as ferramentas utilizadas para esse fim e os resultados obtidos por meio da experiência e as avaliações desenvolvidas pela equipe.

Palavras-chave: Avaliação. Cultura Maker. Criatividade. Ensino remoto. Minicurso.

\section{INTRODUÇÃ̃}

O ano de 2020 ficou marcado pela pandemia de COVID-19. Uma estratégia adotada na maior parte dos estados brasileiros para o enfrentamento da pandemia do novo coronavírus foi o fechamento das escolas públicas, particulares e das universidades. Gerando, assim, um impacto que atingiu, somente na educação básica - de acordo com o último censo disponibilizado pelo INEP -, 47,9 milhões de estudantes (INEP, 2019). Para minimizar os 
efeitos do isolamento social na educação brasileira, o setor tem buscado várias ações de emergência, como por exemplo, a transferência de aulas e outras atividades pedagógicas para o formato on-line (INSTITUTO AYRTON SENNA, 2020).

No dia 17 de março de 2020, o Ministério da Educação (MEC) publicou a portaria $\mathrm{n}^{\circ} 343$ autorizando, em caráter excepcional, a substituição das disciplinas presenciais, em andamento, por aulas que utilizem meios e tecnologias de informação e comunicação, nos limites estabelecidos pela legislação em vigor, por instituições de educação superior integrantes do sistema federal de ensino, pelo período de até 30 dias, prorrogáveis, a depender de orientação do Ministério da Saúde e dos órgãos de saúde estaduais, municipais e distrital. Posteriormente, essa portaria foi prorrogada até o dia 31 de dezembro. Essa paralisação das atividades presenciais afetou também os trabalhos de pesquisa e extensão, fazendo com que muitos estudantes de iniciação científica, sobretudo, nas atividades que dependiam de experimentação em laboratórios tivessem que paralisar seus trabalhos totalmente. Outros continuaram seus trabalhos em home office e em alguns casos foi necessário reformular o planejamento dos projetos a fim de adaptar as ações de acordo com a nova realidade.

O Mundo Maker é um projeto de extensão criado no CEFET-MG, unidade Leopoldina, para divulgar e fomentar ferramentas de desenvolvimento tecnológico utilizando a Cultura Maker em escolas de ensino fundamental e médio da rede pública. $\mathrm{O}$ projeto teve início em novembro de 2019, e tinha como proposta inicial ofertar minicursos presenciais para os estudantes dessas escolas, de forma a permitir o desenvolvimento de pequenos projetos que envolvessem as temáticas de sustentabilidade e eficiência energética. Porém, com a chegada da pandemia de COVID-19 e a suspensão das aulas presenciais, a equipe do projeto Mundo Maker precisou se reestruturar.

Este trabalho apresenta um estudo de caso acerca do projeto de extensão Mundo Maker, mostrando as ações tomadas pelos integrantes do projeto e a forma como os trabalhos foram realizados para viabilizar a conclusão dos objetivos do Mundo Maker para o ano de 2020. A pandemia de COVID-19 representa um momento singular na história da humanidade, mas certamente o desenvolvimento e utilização de novas tecnologias, as metodologias adotadas e os resultados obtidos durante esse período excepcional, apresentam-se como uma nova forma pela qual a educação tem se reinventado para vencer barreiras, além da preparação para enfrentamentos semelhantes no futuro.

O restante deste artigo está organizado da seguinte forma: na Seção 2 é apresentado um breve referencial teórico acerca da Cultura Maker. A Seção 3 detalha a metodologia empregada para a aplicação dessa cultura no decorrer do projeto e considerando o cenário de pandemia. Na Seção 4 tem-se os principais resultados proporcionados pelo projeto. E, por fim, na Seção 5 são expostas as conclusões do trabalho.

\section{CULTURA MAKER}

A Cultura Maker, para Silvano (2018), explora a criatividade, o trabalho em equipe e a capacidade de inovação de jovens e adultos na construção de projetos, desenvolvendo assim, a comunicação, colaboração e resolução de problemas.

O físico e escritor Chris Anderson, em seu livro "The Long Tail” (ANDERSON, 2006), descreve a Cultura Maker como a nova revolução industrial. Segundo ele, o poder saiu das indústrias e foi para a mão do consumidor. Isso pelo fato de o conhecimento não estar mais restrito para alguns, mas, agora, todos tem acesso e capacidade para desenvolver qualquer produto. 
A Cultura Maker origina-se do movimento denominado DIY - Do It Yourself, termo que traduzido para o português significa "faça você mesmo". Conforme Samagaia (2015), esse movimento consiste em grupos de indivíduos que se organizam para suportar mutuamente o desenvolvimento de projetos dos envolvidos.

Segundo Convex, (2019), foi no ano de 2005 que o Movimento Maker se consolidou de fato na cultura, inicialmente nos Estados Unidos, através da criação da Revista Make, fundada por Dale Dougherty. Essa revista tem como objetivo a divulgação de projetos makers, ou seja, o desenvolvimento de protótipos englobando as mais diversas áreas do conhecimento, que originalmente são complexos e de um custo muitas vezes elevado, por um baixo custo e alta eficiência.

Há ainda, a Maker Faire, organizada pela primeira vez em 2006, com o intuito de reunir pessoas de todas as idades que tenham interesse por tecnologia, artesanato, educação e amantes da ciência no geral. Nela, os fabricantes podem apresentar seus projetos e adquirir novos conhecimentos.

Segundo Draft (2016), a Cultura Maker chegou definitivamente no Brasil em 2015, com a criação do seu primeiro laboratório de fabricação, popularmente conhecido como Fab Lab. O Fab Lab SP, localizado na Faculdade de Arquitetura e Urbanismo da Universidade de São Paulo (FAU-USP) foi equipado com os mais variados tipos de ferramentas para fomentar a criatividade dos indivíduos que ali se encontram. Atualmente, há 30 Fab Labs no Brasil, em 10 estados (FABLABS, 2020) e, segundo Silvano (2018), esses laboratórios têm o intuito de proporcionar um ambiente de construção, análises pessoais, discussões em grupo e autoavaliação de resultados.

Para Brockveld et al. (2017), em um ambiente de desenvolvimento Maker, o professor deve deixar a posição de detentor do conhecimento e guiar os estudantes no desenvolvimento do seu próprio conhecimento. O educador e o aluno devem aprender a aprender. Corroborando com essa ideologia, Almeida et al. (2018), aponta que práticas no modelo DIY estimulam o interesse dos discentes, já que propicia a utilização de conhecimentos teóricos na elaboração de projetos, estimulando assim, a criatividade dos envolvidos.

De acordo com Moura et al. (2019), os graduandos dos cursos de engenharia almejam uma formação técnica majoritariamente nas áreas tecnológicas e de ciências exatas, mas, essa formação em conjunto com atividades que façam uso da criatividade, proporcionam o desenvolvimento de competências mais amplas, humanísticas e sociais. Assim, o projeto de extensão citado neste trabalho apresenta-se como uma forma de permitir aos estudantes de Engenharia de Controle e Automação e Engenharia de Computação, o contato direto com estes tipos de atividades.

\section{METODOLOGIA}

Desde o ano de 2019, a equipe do projeto Mundo Maker possuía um planejamento completo de todas as atividades que deveriam ocorrer ao longo do ano de 2020, sendo que algumas dessas atividades, inclusive, já estavam em andamento. Uma das principais atividades a serem realizadas consistia em ofertar minicursos presenciais que exploram conceitos de lógica, programação básica e ciência, utilizando uma abordagem DIY para alunos do Ensino Fundamental II e Ensino Médio. Assim sendo, foram realizadas quatro palestras em diferentes escolas da região, com o intuito de apresentar a Cultura Maker e divulgar os dois minicursos que seriam ofertados: um que utilizava como plataforma base o Arduino e outro que tinha como foco a plataforma MIT App Inventor. A Figura 1 mostra uma dessas palestras realizada na Escola Estadual Professor Botelho Reis, em Leopoldina. 
Figura 1 - Palestra realizada na Escola Estadual Professor Botelho Reis, Leopoldina (MG)

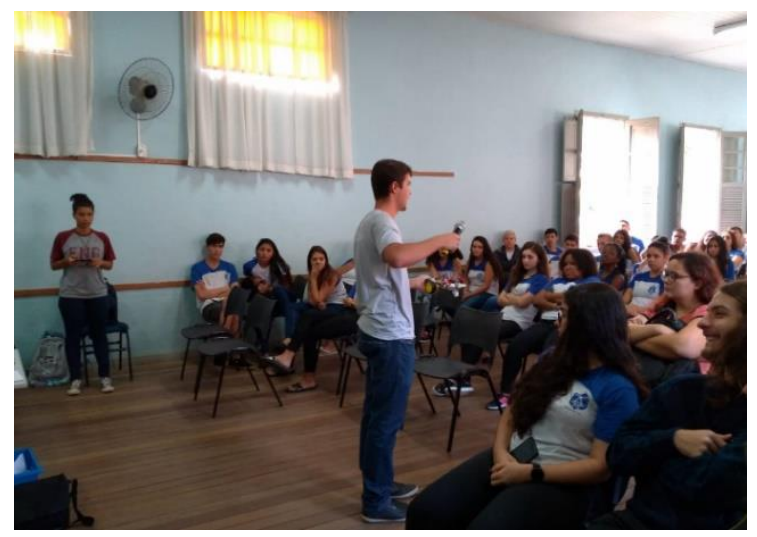

Fonte: Arquivos do Mundo Maker

O Arduino é uma plataforma eletrônica de código aberto baseada em hardware e software fáceis de usar. As placas do Arduino são microcontroladores monoprogramáveis que podem receber informações de sensores, processar essas informações e atuar em saídas como LEDs ou motores (Arduino, 2020).

O MIT App Inventor é uma plataforma de desenvolvimento intuitivo e visual na qual até mesmo crianças conseguem construir aplicativos totalmente funcionais para smartphones e tablets que utilizam o sistema operacional Android. Desenvolvido pela Google e mantido, atualmente, pelo Massachusetts Institute of Technology, o MIT App Inventor possui linguagem de programação baseada em blocos e ferramentas práticas como botões e caixas de texto que facilitam a construção das telas do aplicativo simplificando muito o desenvolvimento de projetos (MIT App Inventor, 2020).

Os minicursos previam um período de capacitação em que os ministrantes ensinariam os conceitos básicos de cada plataforma, usando uma abordagem DIY. Posteriormente seria proposto aos participantes desenvolverem algum projeto relacionado às temáticas de sustentabilidade e eficiência energética. Esses projetos seriam apresentados em uma exposição organizada pela equipe envolvida no projeto Mundo Maker.

A declaração de pandemia de COVID-19 e a suspensão das atividades escolares no formato presencial fez com que o planejamento inicial tivesse que ser adaptado à nova realidade. O projeto de extensão, que conta hoje com 15 estudantes dos cursos de Engenharia de Controle e Automação e Engenharia de Computação e com 3 professores coordenadores do CEFET-MG unidade de Leopoldina, precisou direcionar as atividades de toda a equipe para o formato on-line, através de home office, desde o trabalho envolvendo a divulgação das ações através das mídias sociais do projeto, até a confecção dos materiais didáticos e a própria realização dos minicursos.

Diante do cenário de isolamento social, a equipe adotou de forma bem definida os planos de trabalho individuais, distribuindo de forma igualitária as tarefas a serem cumpridas. Além dessa divisão, foram adotadas reuniões realizadas semanalmente por meio de videoconferência, todas registradas em ATA e devidamente assinada por todos os participantes, a caráter de formalização das decisões.

No decorrer destas reuniões, ocorreram discussões acerca da necessidade da formulação de uma ementa para cada um dos cursos, dessa forma norteando tanto o ministrante do curso, quanto os alunos. Inicialmente, havia a previsão do retorno das atividades presenciais no 
segundo semestre, por isso, ficou definido que os cursos teriam uma carga horária de 22 horas, distribuída em 11 encontros de 2 horas. Então, com essa base, foram propostas as ementas e devidamente aprovadas.

Uma das principais decisões tomadas durante as reuniões semanais foi a que os cursos a serem ofertados seriam ministrados no formato de um ensino remoto, iniciando assim, as adversidades de implementação, haja vista que não seria apenas ministrar o mesmo conteúdo previsto inicialmente, mas sim, adequar a uma filosofia de ensino diferente da que havia sido pensada, tendo como foco não perder a principal característica dos cursos que é a possibilidade de oferecer ao aluno a oportunidade de 'experimentar as tecnologias e desenvolver pequenos projetos com as próprias mãos'.

O primeiro passo de adaptação necessário foi avaliar e definir as ementas. As aulas, que inicialmente estavam previstas para possuírem 2 horas de duração no formato presencial, foram reduzidas para 1 hora, pois no formato on-line, a equipe chegou à conclusão que o aluno, em sua residência, estaria exposto a diversos imprevistos, como queda de conexão com a internet, queda de energia, eventuais distrações, dentre outras, e com essa redução, a probabilidade de dispersão seria menor. Quanto ao número de encontros, foi definido que seriam para 6 aulas semanais.

Além disso, surgiu a ideia de oferecer uma terceira opção de minicurso, intitulado "Maker em Casa", que não faria uso de uma plataforma tecnológica, sendo necessário para o acompanhamento das aulas apenas um smartphone com acesso à internet. Sua criação partiu do intuito de explorar ainda mais o atual momento de isolamento social para estimular a criatividade dos envolvidos, que é um dos principais pilares da Cultura Maker. Nele, os alunos também poderiam praticar o DIY, porém, construindo protótipos utilizando materiais caseiros e visualizando, na prática, conceitos da área de ciências da natureza.

Outra adaptação necessária foi com relação à quantidade de alunos nos cursos. No planejamento para os cursos presenciais, este número estava limitado ao espaço físico dos laboratórios do campus Leopoldina do CEFET-MG. O novo desafio levantado pela equipe era a questão do acompanhamento individual dos participantes por parte do ministrante, de forma a não comprometer a qualidade do aprendizado do conteúdo. Por isso, ficou definido um limite de 10 alunos em cada turma.

Em seguida, foi observada a necessidade de elaborar um material didático pautado na ementa, com o intuito de direcionar o ministrante e facilitar o estudo dos alunos. Assim, foi definido para cada um dos 3 cursos, um responsável por elaborar esse material. Ele era composto por uma apresentação em slides ou por um documento de texto descrevendo o passo-a-passo das práticas e conteúdos teóricos. Antes do início de cada aula, esse material era enviado para o e-mail dos alunos.

Os cursos do projeto de extensão Mundo Maker foram todos ministrados pela plataforma de reuniões e videochamadas Google Meet. As salas contaram com a presença do ministrante e demais membros da equipe do projeto para direcionar eventuais dúvidas, registrar a presença dos alunos, avaliar a efetividade do material de apoio e observar a participação do público-alvo durante as aulas.

Ao final de cada encontro foram endereçadas, aos participantes, mensagens pedindo algumas respostas sobre a visão deles quanto aos conteúdos abordados, aplicabilidade do conhecimento adquirido, críticas e/ou elogios quanto à forma de condução do curso e o levantamento de dificuldades de acompanhamento. Estes pareceres não eram obrigatórios e poderiam ser dados por meio de áudio, vídeo ou texto. Com isso, era esperado um acompanhamento da construção do conhecimento, o diagnóstico do processo de ensino e a identificação de eventuais dificuldades e dúvidas subsequentes aos términos das aulas. 
Os três minicursos desenvolvidos foram conduzidos de forma bem parecida em termos de carga horária e objetivos por aula, distinguindo apenas a plataforma utilizada. Nos casos dos minicursos de MIT App Inventor e Arduino, os ministrantes compartilhavam suas telas explicando os conteúdos e direcionando sobre realizar as atividades práticas. Enquanto no minicurso Maker em Casa, o ministrante demonstrava os procedimentos por meio da sua webcam. O roteiro e ferramentas utilizadas serão detalhadas nos tópicos a seguir.

\subsection{Minicurso de Arduino}

O minicurso de Arduino ocorreu às sextas-feiras, entre os dias 12 de junho e 17 de julho de 2020, com o apoio da plataforma Tinkercad - plataforma on-line e gratuita de desenhos de modelos 3D e simulações de circuitos elétricos desenvolvida pela Autodesk (TINKERCAD, 2020). A razão da utilização desta ferramenta foi a possibilidade de simulação dos circuitos desenvolvidos com a placa de prototipagem Arduino, bem como a programação do microcontrolador da mesma.

Os conteúdos abordados consistiram em entrada digital, saída digital, entrada analógica e escrita analógica. Estes foram transmitidos de forma prática, com a construção conjunta do ministrante do curso e os alunos participantes. A Tabela 1 mostra as atividades práticas abordadas durante as lições, de forma a permitir a realização do $D I Y$ pelos participantes.

Tabela 1 - Práticas desenvolvidas durante o minicurso de Arduino

\begin{tabular}{c|c|}
\hline Prática & Conteúdos Abordados \\
\hline Piscar LED & Saída Digital \\
\hline Botão Controlando LED & Entrada e Saída Digital \\
\hline Simulando um Semáforo & Entrada Digital \\
\hline Acionando Buzzer + LED & Saída Digital \\
\hline Leitura de Sensor de Distância & Entrada e Saída Digital \\
\hline Desenvolvimento de um Sensor de Estacionamento & Entrada e Saída Digital \\
\hline Acionamento de um motor com PWM & Escrita analógica \\
\hline Fade com Arduino & Entrada e Escrita Analógica \\
\hline Liga-Desliga com LDR & Entrada e Escrita Analógica \\
\hline Fonte: Autoria própria &
\end{tabular}

\subsection{Minicurso de MIT App Inventor}

O minicurso de MIT App Inventor ocorreu às quartas-feiras, entre os dias 10 de junho e 15 de julho de 2020. O próprio site do MIT App Inventor oferece uma plataforma gratuita de desenvolvimento web, sem a necessidade de downloads. Para participar do curso o estudante precisava ter apenas um computador com acesso à internet, um celular com sistema operacional Android para poder testar os aplicativos e um e-mail vinculado à Google. Para testar os aplicativos utilizou-se o AI assistent, por isso já na primeira aula foi solicitado aos estudantes que instalassem o aplicativo MIT AI2 Companion nos celulares, para testar os aplicativos criados durante as aulas. A Tabela 2 mostra as atividades práticas abordadas durante as lições. 
"Os desafios para formar hoje o engenheiro do amanhã"

Tabela 2 - Práticas desenvolvidas durante o minicurso de MIT App Inventor

\begin{tabular}{c|c|}
\hline Prática & Conteúdos Abordados \\
\hline Olá Mundo & Acionamento de botões e manipulação de textos \\
\hline Tela Login & Lógicas "Se", "senão", "e" e "ou” \\
\hline Calculadora & Manipulação de variáveis e elementos matemáticos \\
\hline Animais App & Utilização de mídias digitais \\
\hline Paint & Utilização de sensores \\
\hline Me Leva & Utilização de todo o conteúdo abordado \\
\hline \multicolumn{2}{|c|}{ Fonte: Autoria própria }
\end{tabular}

\subsection{Minicurso Maker em Casa}

O minicurso de Maker em Casa ocorreu aos sábados, entre os dias 13 de junho de 2020 e 18 de julho de 2020. E, como citado anteriormente, ele foi criado posteriormente aos demais minicursos ofertados pelo Mundo Maker, devido à necessidade de explorar o momento de adversidade enfrentado. Dessa forma, além de proporcionar o desenvolvimento Maker, era incentivado também uma das temáticas do projeto: sustentabilidade. Para participar desse minicurso, os alunos deveriam ter apenas um smartphone ou computador com acesso à internet, microfone e câmera, além de providenciar os materiais a serem utilizados na aula. A lista de materiais era enviada com 7 dias de antecedência, para facilitar a organização dos alunos ou a preparação / aquisição destes materiais, quando necessário.

Entre os conteúdos ministrados estavam conceitos de eletroquímica, óptica, elétrica, cinemática e conservação de energia. Todos utilizando materiais de fácil acesso e com a ação conjunta do ministrante do curso e os participantes construindo as práticas. As atividades práticas abordadas durante as lições foram Pilha de Daniel, Projetor de Celular, Máquina de Choque, Carrinho que Anda sem Pilha, Catapulta.

\section{RESULTADOS E DISCUSSÕES}

As palestras de exposição do projeto Mundo Maker nas escolas, ocorridas nos meses de fevereiro e março de 2020, atingiram um público de cerca de 500 estudantes. E, ainda que estes não tenham participado de demais atividades no projeto, as palestras tiveram um papel importante de conscientização e envolvimento dos alunos com as temáticas de sustentabilidade e eficiência energética.

Os minicursos desenvolvidos contaram com 57 alunos selecionados. Ao fim da carga horária prevista, um total de 70,17\% (40 alunos) recebeu um certificado de participação. Para ocorrer a certificação, o participante deveria possuir $75 \%$ de presença nas aulas.

Os pareceres não-obrigatórios, recebidos dos participantes, possibilitaram à organização do projeto realizar correções na forma de condução do curso de forma rápida.

Com o formulário final de avaliação, pôde-se caracterizar a satisfação dos participantes quanto a metodologia aplicada no curso. A amostragem obtida foi de 34 respostas, que representa $85 \%$ da população total de alunos certificados. A Figura 2-a mostra a percepção dos alunos quanto ao impacto que os cursos geraram em sua criatividade, cerca de $67,7 \%$ acreditam que sua criatividade melhorou muito ou extremamente. Enquanto a Figura 2-b apresenta a avaliação dos participantes sobre a melhoria do raciocínio lógico através das práticas desenvolvidas, com $67,6 \%$ da amostra afirmando que esta habilidade melhorou muito ou extremamente. 


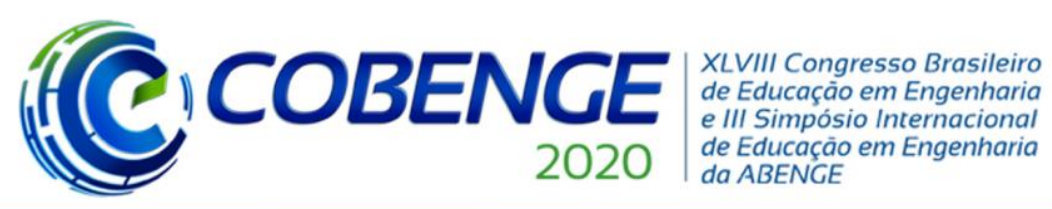

"Os desafios para formar hoje o engenheiro do amanhã"
$\mathrm{O}$ a $\mathrm{O3}$ de dezembro Evento On-line

Figura 2 - Avaliação dos participantes. a) sobre sua criatividade; b) sobre seu raciocínio lógico

O quanto sua criatividade melhorou após o(s) curso(s)?

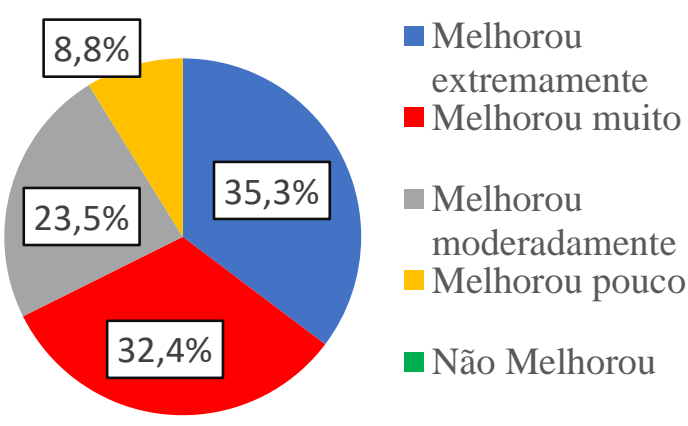

a)

Fonte: Autoria própria
O quanto seu raciocínio lógico melhorou após o(s) curso(s)?

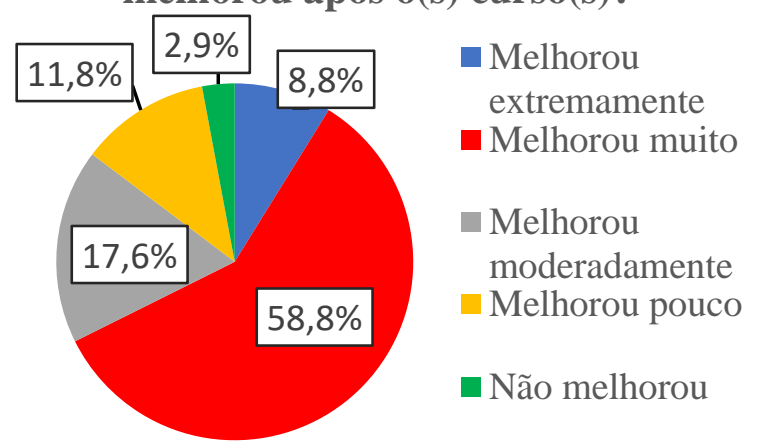

b)

Com base nos dados apresentados na Figura 3, é possível notar que a generalidade dos participantes dos cursos, cerca de $97 \%$ do total, avalia que conseguiu assimilar mais do que $60 \%$ dos conteúdos.

Figura 3 - Avaliação dos participantes sobre assimilação de conteúdo

\begin{tabular}{|l|}
$\begin{array}{c}\text { Como você avaliaria sua taxa de aprendizado dos } \\
\text { conhecimentos expostos? }\end{array}$ \\
$\square$ De 81 a $100 \%$ \\
$\square$ De 61 a $80 \%$ \\
$\square$ De 41 a $60 \%$ \\
De 21 a $40 \%$ \\
$\square$ De 0 a $20 \%$
\end{tabular}

Fonte: Autoria própria

\section{CONSIDERAÇÕES FINAIS}

O presente trabalho teve como objetivo apresentar a experiência de ação extensionista no desenvolvimento de aprendizado à distância.

Desde as fases preliminares do projeto, o grupo dedicou-se para discutir acerca da aplicação do ensino baseado nos preceitos da Cultura Maker. Este conhecimento foi atilado 
para que ocorresse a reformulação das metodologias previamente discutidas em metodologias de ensino mediadas por tecnologias.

No cenário on-line, é dificultosa a observância dos participantes para gerar a validação da proposta metodológica. Por isso, optou-se por constatar avanços através do estímulo aos participantes para resolução de problemas em conjunto, debate de ideias e retroalimentar a organização do projeto por meio dos pareceres enviados ao final das aulas.

Os passos seguintes do projeto englobam a consolidação de uma metodologia ativa de avaliação do ensino a distância baseado na Cultura Maker, elaboração de uma nova rodada de cursos e organização de um evento de exposição de projetos criados pelos participantes dos minicursos.

\section{Agradecimentos}

Os autores agradecem ao FNDE, MEC/PET/SESu e a DEDC/CEFET-MG pelo apoio do desenvolvimento do trabalho.

\section{REFERÊNCIAS}

ALMEIDA, Alexandre A.; SILVA, Amós C.; SANTOS, Camila A. M.; SOUZA, Edmar E. P. Espaço Maker nos Anos Finais do Ensino Fundamental: Possibilidades e Desafios Vivenciados por Estudantes de Graduação do Curso de Engenharia. In: VII Congresso Brasileiro de Informática na Educação, 2018, Ceará. Anais. Fortaleza, 2018.

ANDERSON, Chris. The Long Tail. 1ª edição, Estados Unidos: Hyperion, 2006.

ARDUINO, What is Arduino? Disponível em:

https://www.arduino.cc/en/Guide/Introduction. Acesso em: 20 jul. 2020.

BROCKVELD, Marcos V. V; TEIXEIRA, Clarissa S.; Silva, Mônica R. A Cultura Maker em Prol da Inovação: Boas Práticas Voltadas a Sistemas Educacionais. In: XXVII Conferência Anprotec, 2017, Rio de Janeiro. Anais. Rio de Janeiro, 2017.

CONVEX. A história da cultura maker. Disponível em: https://convexnet.com.br/historiada-cultura-maker/. Acesso em: 14 jul. 2020.

FABLABS. Rede Fab Lab Brasil. Disponível em: https://www.fablabs.io/organizations/rede-fab-lab-brasil. Acesso em: 20 jul. 2020.

GINEZI, C. Saiba como funcionam os laboratórios de inovação - fab labs - espalhados pelo Brasil. E como você pode usá-los. Disponível em: https://www.projetodraft.com/saibacomo-funcionam-os-laboratorios-de-inovacao-fab-labs-espalhados-pelo-brasil-e-como-vocepode-usa-los/. Acesso em: 20 jul. 2020.

INSTITUTO AYRTON SENNA. Estudos sobre educação e o impacto da pandemia do coronavírus. Disponível em: https://www.institutoayrtonsenna.org.br/pt-br/socioemocionaispara-crises/estudos-educacao-e-impacto-coronavirus.html. Acesso em: 30 jul. 2020. 
INSTITUTO NACIONAL DE ESTUDOS E PESQUISAS EDUCACIONAIS ANÍSIO TEIXEIRA (INEP), Censo Escolar 2019. Disponível em:

http://portal.inep.gov.br/web/guest/resultados-e-resumos. Acesso em 19 jul. 2020.

MIT APP INVENTOR, About us. Disponível em http://appinventor.mit.edu/about-us. Acesso em: 20 jul. 2020.

MOURA, Daniel B. A.; FONTES, Andrea R. M.; SOUZA, Renato L. R.; PARAVIZO, Esdras. Contribuições da Cultura Maker para o Ensino de Engenharia de Produção no Contexto das Novas Diretrizes Curriculares In: XXXIX Encontro Nacional de Engenharia de Produção, 2019, São Paulo. Anais. Santos, 2019.

SAMAGAIA, Rafaela; NETO, Demétrio D. Educação científica informal no movimento "maker". In: X Encontro Nacional de Pesquisa em Educação em Ciências, 2015, São Paulo. Anais. Águas de Lindóia, 2015.

SILVANO, Jackson. Makerfólio: uma proposta para registros de experiências maker para acompanhamento avaliativo. 2018. 98 f. Tese (Mestrado) - Faculdade de Educação, Universidade do Vale do Itajaí, Itajaí, 2018.

TINKERCAD. Da mente ao projeto em minutos. Disponível em https://www.tinkercad.com/. Acesso em: 19 jul. 2020.

\title{
APPLYING THE MAKER CULTURE IN THE SOCIAL ISOLATION SCENARIO
}

\begin{abstract}
This paper presents a case study about the actions taken by the Extension Project "Mundo Maker" from CEFET-MG, during the social isolation scenario. The Mundo Maker has for goal the popularization of science and technologies associated to the new digital era in society, with focus on students from elementary and high school, following the philosophies of the maker culture. Among the actions of the project, it stands out the realization of minicourses that explore the logic concepts, basics programming and Science, using a practical approach, also building a diversified evaluative monitoring. And, because the COVID-19 pandemic, the Mundo Maker needed to transfer its actions, initially planned to be in a faceto-face format, to an online instruction model. In this paper, it will be explained the origin of this culture that inspired the project, as such as its importance to the teaching integration, not only for the elementary/high school, but also to the graduation. It also presents a report of the methodologies used to offer the mini-courses in a virtual environment with same quality as the face-to-face method, the tools used to do it and the results obtained from experience and assessment developed by the team.
\end{abstract}

Keywords: Evaluation. Maker culture. Creativity. Remote education. Mini-course. 